\title{
The impact of emergency antibiotic administration time on patients with sepsis
}

Saqer M. Althunayyan, MBBS, SBEM, Mohammed A. Aljanoubi, BSc, MSc, Sultan M. Alghadeer, PharmD, BCPS, Musab Z. Alharthi, BSc, Raied N. Alotaibi, BSc, MSc, Abdullab M. Mubarak, BSc, MPH, Abdulaziz M. Almutary, MBBS, SBEM.

\begin{abstract}

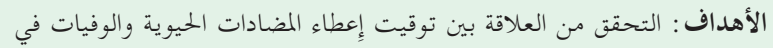

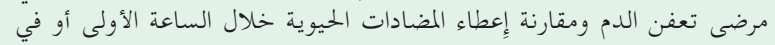
غضون ثلاث ساعات من الفرز في حالات الطوارئ مثار جدل العض في الوسط الطبي. المنهجية : أجريت هذه الدراسة بأثر رجعي، استخدمنا تحليل البيانات الثانوي

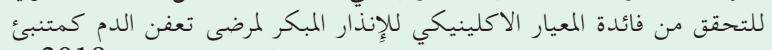

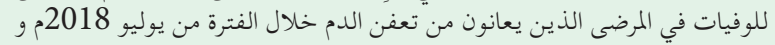

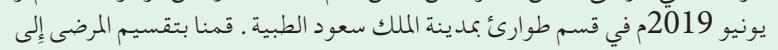

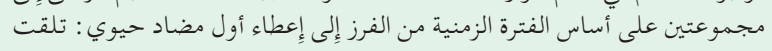

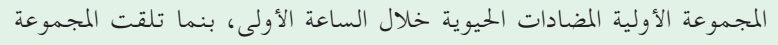

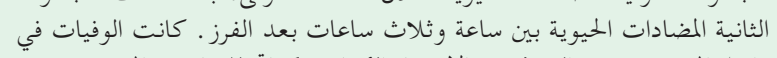

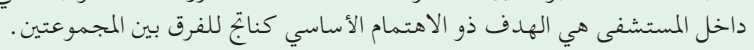

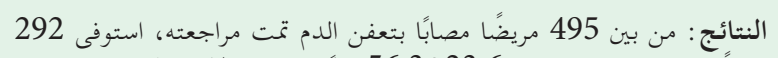

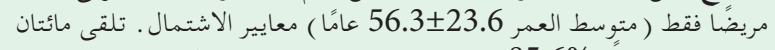

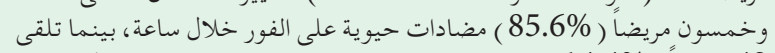

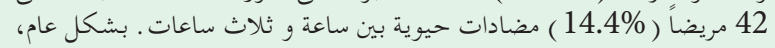

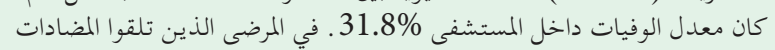

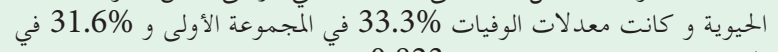
المجموعة الثانية ، وبقيمة احتمالية 0.823.

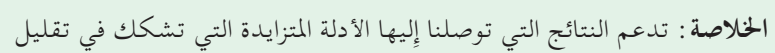

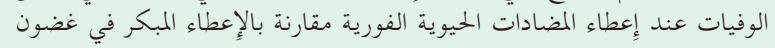

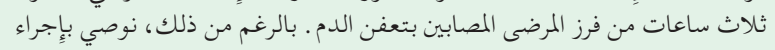

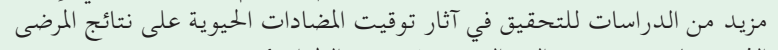
الذين يعانون من تعفن الدم الشّديد في قسم الطوات ارئ.
\end{abstract}

Objectives: To assess the mortality benefits of timely antibiotic treatment of adults present at the emergency department with sepsis and compare one-hour administration and 3-hour administration starting from the time of triage.

Methods: In this retrospective study, we used secondary data analysis to investigate the utility of the National Early Warning Score as a predictor of mortality in sepsis patients between July 2018 and June 2019, at the Emergency Department, King Saud Medical City, Riyadh, Saudi Arabia. The patients were grouped into
2 based on the time interval from triage to the first antibiotic administration: the immediate group received antibiotics within the first hour, and the early group received antibiotics between one and 3 hours. The primary outcome of interest was in-hospital mortality.

Results: Out of 495 septic patients, only 292 patients (mean age of $56.3 \pm 23.6$ years) met the inclusion criteria. Two hundred fifty (85.6\%) patients received antibiotics within one hour of triage (immediate), while $42(14.4 \%)$ patients received antibiotics between one and 3 hours (early). Overall, in-hospital mortality was $31.8 \%$. The mortality rates among patients who received early antibiotic was $31.6 \%$ and who received immediate antibiotic was $33.3 \%$, with a $p$-value of 0.823 .

Conclusion: Our findings did not support immediate antibiotic administration over early administration in patients with sepsis. However, further studies are recommended to investigate the effects of antibiotic timing on the outcome of severe sepsis patients.

Keywords: antibiotics, sepsis, time to antibiotics, infection, emergency antibiotic

Saudi Med J 2021; Vol. 42 (9): 1002-1008 doi: 10.15537/smj.2021.42.9.20210447

From the Department of Trauma (Althunayyan, Alharthi); from the Department of Aviation and Marines (Aljanoubi); from the Department of Basic Sciences (Alotaibi, Mubarak), Prince Sultan College for EMS; from the Department of Clinical Pharmacy (Alghadeer), College of Pharmacy; and from the Emergency Department (Almutary), King Saud University, Riyadh, Kingdom of Saudi Arabia.

Received 7th June 2021. Accepted 12th August 2021

Address correspondence and reprint request to: Dr. Saqer $M$. Althunayyan, Department of Trauma, Prince Sultan College for EMS, King Saud University, Riyadh, Kingdom of Saudi Arabia. E-mail: salthunayyan@ksu.edu.sa

ORCID ID: orcid.org/0000-0001-7994-3412 
Cepsis is an extreme immune response to an infection. The Third International Consensus held in 2016 defined sepsis syndrome as life-threatening organ dysfunction resulting from dysregulated host responses to infection. ${ }^{1,2}$ The impact of sepsis syndrome on healthcare outcomes is significant, as the incidence estimated to be 49 million per year globally. ${ }^{3}$ Almost $26 \%$ of septic patients required intensive care unit (ICU) care. ${ }^{4}$ When considering mortality rate as an ultimate clinical outcome, different reports in the United States indicate the range of $28 \%$ and in Europe the range of $42 \% .^{5}$

To mitigate the burden of sepsis on healthcare outcomes either clinically or financially, multiple approaches and guidelines have been proposed. One of the most popular guidelines for managing sepsis is early goal-directed therapy (EGDT), which focuses mainly on the early administration of antibiotics and early optimization of hemodynamic perfusion and oxygen delivery. ${ }^{6,7}$ Early goal-directed therapy guidelines are divided into 3 hours resuscitation and 6 hours septic shock treatment bundles. ${ }^{1}$ Years later, both bundles were combined into the one-hour bundle in the guidelines of the Surviving Sepsis Campaign (SSC; 2016). ${ }^{8,9}$ Antibiotic administration, as part of EGDT, has always been recommended to be provided early in sepsis management. ${ }^{8}$ The transition to one-hour recommendations for antibiotic administration was based on reports stating that every hour of delay in antibiotic administration is associated with a $7.6 \%$ decline in survival rate. ${ }^{10}$ Such association between death and delay of antibiotic administration has also been reported in multiple studies. ${ }^{11,12}$ Therefore, it was predictable that the most recent SSC recommendations are consistent with such reports and strongly recommend earlier administration of antibiotics (within one hour of the patient's arrival to the emergency department [ED]).

Conversely, multiple reports indicate that selecting the appropriate antibiotics should take precedence over the timing of the administration, as early administration contributes to an increase in antibiotic resistance rates. A prospective observational study for septic cases in the ED found no association between delay in antibiotic administration and increase in length of hospital stay and in-hospital mortality rate. ${ }^{13}$ Another large

Disclosure. Authors have no conflict of interests, and the work was not supported or funded by any drug company. prospective study that enrolled 1,124 patients from different settings (the ED, wards, or the ICU) found no mortality differences between patients who received antibiotics within one to 3 hours from the time of sepsis recognition. ${ }^{14} \mathrm{~A}$ recent meta-analysis that reviewed 33,863 patients with sepsis showed no differences in terms of mortality rate between immediate (within one hour) and early (between one and 3 hours) antibiotic administration. ${ }^{15}$

As the evidence is contradictory, uncertainty on whether antibiotic administration within one hour is superior to 3 hours from the time of ED triage still exists. Moreover, there is a lack of ED studies aimed at investigating the impact of antibiotic administration in sepsis cases on patient outcomes in the Middle East region. Therefore, we aimed to assess the mortality benefits of timely antibiotic treatment of adults present to the ED with sepsis and compare one-hour administration and 3-hour administration starting from the time of triage.

Methods. Data were collected retrospectively between July 2018 and June 2019, from the Emergency Department, King Saud Medical City, Riyadh, Saudi Arabia. The hospital is a government tertiary healthcare facility that had 1,500 beds, $65 \mathrm{ED}$ beds, and $141 \mathrm{ICU}$ beds during the study period, with an estimated 400,000 ED annual visits. This study is a secondary analysis of the data investigating the utility of triage National Early Warning Score (NEWS) as a predictor of mortality in patients with sepsis. ${ }^{16}$ The primary project enrolled all sepsis cases screened in the triaged area. Different levels of NEWS values were examined to identify the best threshold linked to unfavorable clinical outcomes (such as ICU admission, length of stay, and death). All data were kept confidential and not shared with any third party. Because of retrospective observation design of this study, a waiver of informed consent was granted by the IRB in compliance with the Declaration of Helsinki.

Patients were enrolled in the sepsis management protocol according to a NEWS value $>4$, or a clinical sepsis suspect was enrolled by the ED physician who triaged him/her to the high acuity area to start standardized ED sepsis management protocol according to King Saud Medical City's guidelines. The sepsis sheets were completed by the bedside nurses and included triage vitals, lactate levels, and information on antibiotics and cultures, with time for each intervention. Vasopressors were given to hypotensive despite of adequate fluid resuscitation. Antibiotics administered include Piperacillin/Tazobactam (manufactured by SUANFARMA, Madrid, Spain), Ceftriaxone 
(Pfizer Inc, New York, USA) and Vancomycin (Xellia Pharmaceuticals ApS Copenhagen, Denmark)

A total of 495 patients were enrolled in the sepsis management protocol, but 203 patients did not meet the inclusion criteria for several reasons which is pregnancy, less than 18 years old, discharged against medical advice, had a comorbid diagnosis (asthma exacerbation, paroxysmal supraventricular tachycardia, hepatic encephalopathy, and diabetic ketoacidosis), and did not take antibiotics within 3 hours of enrollment or missed the time. The inclusion and exclusion criteria are summarized in Figure 1. Patients included in the study were divided into 2 groups: 1) the immediate group, which received the first IV antibiotics within an hour of triage (from 0 to $60 \mathrm{~min}$ ), and 2) the early group, which received the first antibiotics between one and 3 hours (61 to $180 \mathrm{~min}$ ) after sepsis diagnosis. Time 0 indicates patient triage time, and the endpoint was in-hospital mortality for each group.

Statistical analysis. Stata version 15.1 (StataCorp, College Station, TX, USA) was utilized to analyze the data. Data normality was evaluated using the Shapiro-Wilk test, continuous variables were described using mean and standard deviation, and categorical variables were described as numbers and percentages. The difference between groups was analyzed using a 2 -tailed student's t-test for continuous variables in variables normally distributed and a Chi-square test for categorical variables. For variables that not normally distributed, the median and interquartile ranges are presented, and the Mann-Whitney U-test was used to analyze intergroup differences. Complete case analysis was performed, and statistical significance was determined at $p<0.05$ in all analysis sections. ${ }^{17,18}$

Results. Out of 495 septic patients treated in the ED during the study period, only 292 patients met the inclusion-exclusion criteria for the analysis and 203 patients were excluded (Figure 1). The mean age of $56.3 \pm 23.6$ years and the most common cause of infection was pneumonia $(n=108,37.0 \%)$. Of the 292 patients who received antibiotics, $250(85.6 \%)$ received immediate antibiotics (within the first hour after triage), whereas 42 (14.4\%) were administered early antibiotics (between one and 3 hours) after sepsis diagnosis. Patients who received antibiotics later were older than those who received immediate antibiotics (with a mean age of $61.2 \pm 22.2$ years versus $55.3 \pm 23.8$ years). However, this variability in age between the 2 groups was not statistically significant $(p=0.1754)$. Similarly, there was a non-statistically significant variation between the 2 groups in the number of patients with systolic blood pressure $(\mathrm{SBP})<90 \mathrm{mmHg}(p=0.9804)$ (Table 1$)$.

In our sample, overall, the mortality was $31.8 \%$. In patients who received early antibiotics, mortality was $33.3 \%$, which is slightly higher than that of patients who received immediate antibiotics $(31.6 \%)$, but the

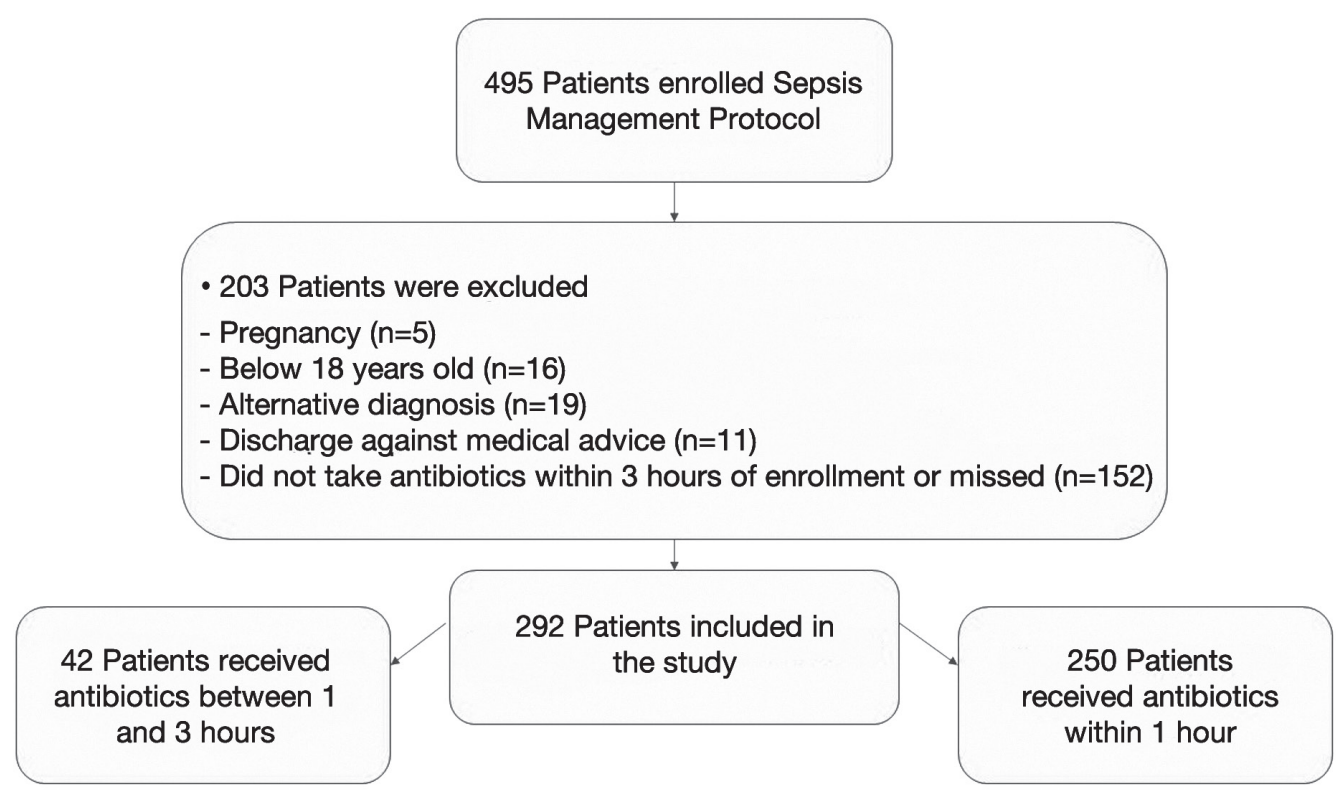

Figure 1 - Flowchart illustrating the inclusion and exclusion criteria. 
$p$-value was not statistically significant for this difference in our cohort $(p=0.8230)$ (Table 1$)$.

The number and distribution of patient comorbidities are shown in Table 1. The most common comorbidities reported in the final cohort were hypertension $(n=101)$ and diabetes mellitus $(n=87)$. Most of those patients received immediate antibiotics.

The most common antibiotic used in the first hour was Piperacillin/Tazobactam (46.4\%), followed by Ceftriaxone (29.6\%). For patients who received antibiotics after one hour, Ceftriaxone was the most commonly used antibiotic (40.5\%), followed by Piperacillin/Tazobactam (28.6\%)(Table 2).

Subgroup analysis for severe hypotensive septic patients with triage SBP $<90 \mathrm{mmHg}$ or $\mathrm{MAP}<65$ $\mathrm{mmHg}$ is shown in Table 3.

Out of the total number of patients, 58 out of 65 patients with SBP $<90$ or MAP $<65$ received immediate

Table 1 - Characteristics of septic patients who received antibiotics within one hour versus those who received antibiotics between one and 3 hours.

\begin{tabular}{|c|c|c|c|c|}
\hline Characteristics & $\begin{array}{l}\text { Overall } \\
\mathrm{N}=292\end{array}$ & $\begin{array}{l}\text { Within } 1 \text { hour } \\
\mathrm{n}=250\end{array}$ & $\begin{array}{l}\text { Between } 1 \text { and } 3 \text { hours } \\
\mathrm{n}=42\end{array}$ & $P$-value \\
\hline Mean age, years $( \pm S D)$ & $56.3(23.6)$ & $55.3(23.8)$ & $61.2(22.2)$ & 0.1754 \\
\hline $\mathrm{SBP}<90 \mathrm{mmHg}$ & $65(22.3)$ & $58(23.2)$ & $7(16.7)$ & 0.9804 \\
\hline Vasopressor use & $39(13.4)$ & $34(13.6)$ & $5(11.9)$ & 0.0893 \\
\hline \multicolumn{5}{|l|}{ Laboratory } \\
\hline Creatinine, mean $( \pm \mathrm{SD})$ & $186.1(224.1)$ & $193.0(233.8)$ & $144.8(148.5)$ & 0.1593 \\
\hline First lactate value, $\mathrm{mmol} / \mathrm{L}$, mean $( \pm \mathrm{SD})$ & $3.4(2.7)$ & $3.5(2.7)$ & $3.0(2.5)$ & 0.2937 \\
\hline Leucocytosis (WBC $>11)$ or leukopenia $(<4)$ & $161(55.1)$ & $142(56.8)$ & $19(45.2)$ & 0.1633 \\
\hline Hospital mortality & $93(31.8)$ & $79(31.6)$ & $14(33.3)$ & 0.8230 \\
\hline Any comorbidities & $145(49.6)$ & $123(49.2)$ & $22(52.4)$ & 0.7028 \\
\hline Diabetes mellitus & $87(29.8)$ & $86(34.4)$ & $1(2.4)$ & $<0.0001$ \\
\hline Hypertension & $101(34.6)$ & $99(39.6)$ & $2(4.8)$ & $<0.0001$ \\
\hline Congestive heart failure & $12(4.1)$ & $7(2.8)$ & $5(11.0)$ & 0.0059 \\
\hline Kidney failure & $21(7.2)$ & $19(7.6)$ & $2(4.8)$ & 0.5100 \\
\hline Oncology & $19(6.5)$ & $14(5.6)$ & $5(11.9)$ & 0.1253 \\
\hline \multicolumn{5}{|l|}{ Source of infection } \\
\hline Pneumonia empyema & $108(37.0)$ & $106(42.4)$ & $2(4.8)$ & $<0.0001$ \\
\hline Urinary tract infection & $29(9.9)$ & $23(9.2)$ & $6(14.3)$ & 0.3079 \\
\hline Acute abdominal infection & $8(2.7)$ & $7(2.8)$ & $1(2.4)$ & 0.8776 \\
\hline Meningitis & $14(4.8)$ & $8(3.2)$ & $6(14.3)$ & 0.0019 \\
\hline Skin/soft tissue infection & $32(11)$ & $26(10.4)$ & $6(14.3)$ & 0.4557 \\
\hline Bone/joint infection & $7(2.4)$ & $1(0.4)$ & $6(14.3)$ & $<0.0001$ \\
\hline Wound infection & $7(2.4)$ & $1(0.4)$ & $6(14.3)$ & $<0.0001$ \\
\hline Blood stream catheter infection & $7(2.4)$ & $1(0.4)$ & $6(14.3)$ & $<0.0001$ \\
\hline
\end{tabular}

Table 2 - Type of antibiotic used in patients within one hour versus those who received antibiotics between one and 3 hours.

\begin{tabular}{|c|c|c|c|c|}
\hline $\begin{array}{l}\text { Most common } \\
\text { antibioticm, } \mathrm{n}(\%)\end{array}$ & $\begin{array}{l}\text { Overall } \\
\mathrm{N}=292\end{array}$ & $\begin{array}{l}\text { Within one hour } \\
\mathrm{n}=250\end{array}$ & $\begin{array}{l}\text { Between } 1 \text { and } 3 \text { hours } \\
n=42\end{array}$ & $P$-value \\
\hline First & $\begin{array}{c}\text { Piperacillin/Tazobactam } \\
188(42.3)\end{array}$ & $\begin{array}{l}\text { Piperacillin/ } \\
\text { Tazobactam } \\
116(46.4)\end{array}$ & $\begin{array}{l}\text { Ceftriaxone } \\
17(40.5)\end{array}$ & 0.4756 \\
\hline Second & $\begin{array}{l}\text { Ceftriaxone } \\
137(30.9)\end{array}$ & $\begin{array}{l}\text { Ceftriaxone } \\
74(29.6)\end{array}$ & $\begin{array}{c}\text { Piperacillin/Tazobactam } \\
12(28.57)\end{array}$ & 0.8923 \\
\hline Third & $\begin{array}{l}\text { Vancomycin } \\
45(10.1)\end{array}$ & $\begin{array}{l}\text { Vancomycin } \\
31(12.4)\end{array}$ & Vancomycin $(5 ; 11.9)$ & 0.9280 \\
\hline
\end{tabular}


Table 3 - Characteristics of severe septic patients with systolic blood pressure $<90 \mathrm{mmHg}$ or mean arterial pressure $<65 \mathrm{mmHg}$ who received antibiotics within one hour versus those who received antibiotics between 1 and 3 hours $(\mathrm{N}=65)$.

\begin{tabular}{lccc}
\hline Characteristics of patients & $\begin{array}{c}\text { Within one hour } \\
\mathbf{n}=58\end{array}$ & $\begin{array}{c}\text { Between } \mathbf{1} \text { and } 3 \text { hours } \\
\mathbf{n}=7\end{array}$ & $P$-value \\
\hline Age & $59.86(21.65)$ & $67.8(21.58)$ & 0.5731 \\
Any comorbidities & $33(56.9)$ & $5(71.4)$ & 0.4611 \\
Diabetes mellitus & $22(37.9)$ & $2(28.6)$ & 0.463 \\
Hypertension & $25(43.1)$ & $2(28.6)$ & 0.309 \\
Congestive heart failure & 0 & 0 & NA \\
Kidney failure & $4(6.9)$ & 0 & NA \\
Oncology & $14(24.1)$ & $3(42.8)$ & 0.287 \\
Source of infection & & & \\
Pneumonia empyema & $9(15.5)$ & $1(14.3)$ & 0.932 \\
Urinary tract infection & $5(8.6)$ & 0 & NA \\
Acute abdominal infection & $2(3.4)$ & 0 & NA \\
Meningitis & $12(20.7)$ & $1(14.3)$ & 0.689 \\
Skin/soft tissue infection & $1(1.7)$ & 0 & NA \\
Bone/joint infection & $1(1.7)$ & 0 & NA \\
Wound infection & $1(1.7)$ & 0 & NA \\
Vasopressor & $18(31.0)$ & $2(28.6)$ & 0.894 \\
Mortality & $22(37.9)$ & $3(42.8)$ & 0.800 \\
\hline
\end{tabular}

Values are presented as numbers and percentages $(\%)$.

antibiotics within the first hour. Only 7 patients with SBP $<90$ or MAP $<65$ received early antibiotics. In our analysis we did not found a statistical significant deference in the mortality of both groups. This low number of observations in subgroups makes it difficult to produce a robust statistical analysis to draw an appropriate conclusion. However, Figure 2 illustrate the mortality rate among both groups for sepsis and severe sepsis patients.

Discussion. The 2018 updated SSC bundle recommends providing antibiotics within the first hour of sepsis identification. ${ }^{8}$ Several well-recognized medical societies are against the new recommendation of a one-hour antibiotic bundle for sepsis management. The European Society of Emergency Medicine criticizes the level of evidence presented by the SSC, the ignorance of a realistic acute-care environment, and the absence of patient-related harmful considerations; thus, it has recommended against implementing the one-hour bundle. ${ }^{19}$ Even the Society of Critical Care Medicine mentioned that the interventions might not be necessarily to be completed in the first hour. ${ }^{20}$ Furthermore, the new SSC guidelines was not endorsed by the Infectious Diseases Society of America to avoid unnecessary antibiotic utilization. ${ }^{21}$ These concerns

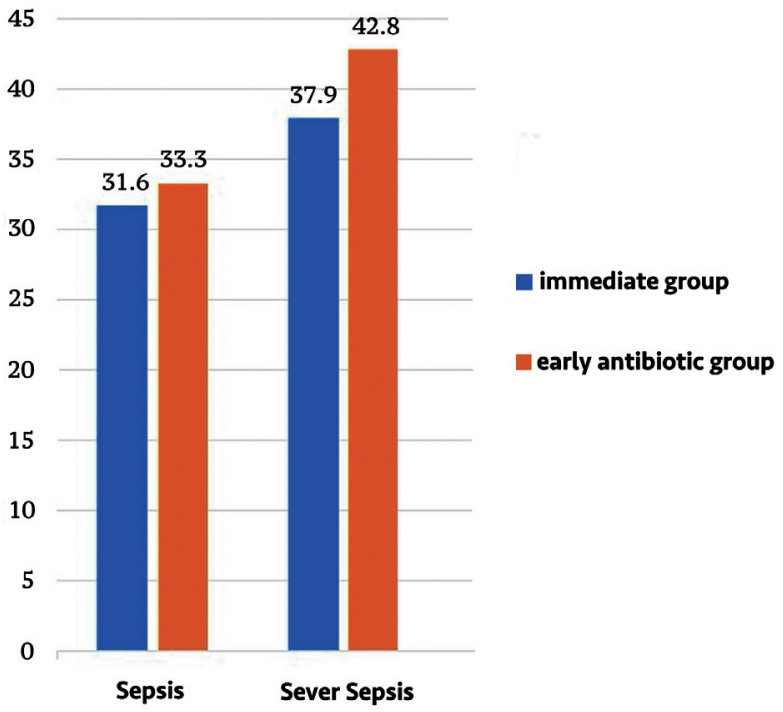

Figure 2 - Illustrate the mortality rate among both groups for sepsis and severe sepsis patients.

raised a question of whether the one-hour antibiotic administration compared to the 3-hour administration would significantly impact mortality in patients with sepsis.

The study results show that $85.6 \%$ of the patients with sepsis received immediate antibiotics within one 
hour of undergoing triage because of adherence to the hospital sepsis protocol, whereas $14.4 \%$ received antibiotics early (between one and 3 hours). Our findings reveal no significant benefits of immediate antibiotic administration (31.6\%) compared to early administration (33.3\%) regarding mortality. According to a recent meta-analysis that included 13 studies from different countries and 33,863 participants, there were no mortality benefits (odds ratio [OR] 1.09; 95\% confidence interval [CI] 0.98-1.21) between immediate (within one hour) and delayed (between one and 3 hours) antibiotic administration. ${ }^{15}$ Additionally, in another small meta-analysis, the mortality rates for immediate (within one hour) versus delayed (between one and 3 hours) antibiotic administration were similar, (36.6\% versus $36.8 \%) .{ }^{22}$ Besides the literature evidence regarding the debate on the first-hour administration of an antibiotic, the feasibility of giving the appropriate antibiotics within the first hour of sepsis in the emergency is another clinical dilemma and is not always practical. ${ }^{10}$ However, one-hour versus 3-hour antibiotic administration in patients with severe sepsis or septic shock shows conflicting results.

Differences in outcome could be attributed to patient factors concerning multiple aspects. Generally, patients who are sicker or patients who are more vulnerable to severe infection could receive antibiotics earlier. However, among our study participants with severe sepsis/septic shock presented with hypotension, there were no significant differences in mortality rates between patients who received antibiotics within the first hour and those who received antibiotics later. Since only 65 patients presented with hypotension, it is difficult to draw definitive conclusions on the timing of antibiotic administration. Nevertheless, consistent with our findings, a study conducted among 1,184 patients with severe sepsis or septic shock in Japan ${ }^{14}$ did not find that early (within one hour) antibiotic administration causes a reduction in hospital mortality (OR 0.999; $p=0.152$ ). Sterling et $\mathrm{al}^{22}$ reported similar results in a small meta-analysis.

In contrast, a study considered time to antibiotic administration as the key element for the progression from severe sepsis to septic shock (OR 1.06; 95\% CI 1.05-1.08) and for in-hospital mortality. ${ }^{24}$ Negative mortality outcomes have also been associated with the length of time of antibiotic administration in patients with severe sepsis or septic shock, as described in a structured review article. ${ }^{25}$ Paradoxically, according to the most recent large meta-analysis conducted by Rothrock et al, ${ }^{15}$ the mortality rate of severely septic patients was higher in patients who received antibiotics within the first hour compared to patients received antibiotics between one and 3 hours. These results were also described in the study in Japan, which revealed the mortality rate to be the highest within the first hour group among patients with severe sepsis or septic shock (mortality rate $=28.7 \%, 95 \%$ CI 23.3-34.1). ${ }^{14}$ Therefore, we highly recommend conducting a large, randomized trial to assess the effects of antibiotic timing on patients with severe sepsis in the ED.

Study limitations. First, it is a single center study with a relatively small participant size, and an uneven distribution between the 2 comparative groups. Due to the small sample size, we were not able to match cases to controls by all possible confounders, such as comorbidities. However, the data of both cases and controls were included from the same hospital over the same period, and there was no significant variation by age, leaving both groups at relatively similar risks of comorbidities. Second, the retrospective nature of the study and poor documentation led to some missing information that prevented us from assessing the appropriateness of antibiotic selection and dosages. Lastly, we did not study a specific time short-term outcome, as confounder's effect might increase with the lengths of stay.

It is known that the most recent Surviving Sepsis Campaign (SSC; 2018) guidelines strongly recommend earlier administration of antibiotics (within the first hour of sepsis identification) during the sepsis management ${ }^{8}$ since there is an association between death and delay of antibiotic administration (beyond one hour). ${ }^{11,12,23 \text {, }}$ ${ }^{24}$ However, our study along with other recent studies found no mortality advantage of immediate antibiotic administration over early administration within 3 hours of triaging patients with sepsis. ${ }^{13-15,22}$ Additionally, a concern on the inappropriate use of antibiotics and possible shift from patient-centered care to hospital quality-standard adherence exists since the immediate administration of antibiotics (part of the one-hour bundle) is considered an unfavorable recommendation.

In conclusion, regarding mortality in our study, we did not find a significant preference of immediate antibiotic administration over early administration in patients with sepsis. However, we recommend conducting a large randomized trial to investigate the effects of antibiotic timing on the outcome of patients with severe sepsis in the ED.

Acknowledgment. Our appreciation goes to the Deanship of Scientific Research, King Saud University, Riyadh, Saudi Arabia for supporting this work through research group number $R G$ 1441-518. We would like to thank American Manuscript Editors (www.americanmanuscripteditors.com) for English language editing. 


\section{References}

1. Singer M, Deutschman CS, Seymour CW, Shankar-Hari M, Annane D, Bauer M, et al. The third international consensus definitions for sepsis and septic shock (Sepsis-3). JAMA 2016; 315: 801-810.

2. Saria S, Henry KE. Too many definitions of sepsis: can machine learning leverage the electronic health record to increase accuracy and bring consensus? Crit Care Med 2020; 48: 137-141.

3. Rudd KE, Johnson SC, Agesa KM, Shackelford KA, Tsoi D, Kievlan DR, et al. Global, regional, and national sepsis incidence and mortality, 1990-2017: analysis for the global burden of disease study. Lancet 2020; 395: 200-211.

4. Sakr Y, Jaschinski U, Wittebole X, Szakmany T, Lipman J, Namendys-Silva SA, et al., editors. Sepsis in intensive care unit patients: worldwide data from the intensive care over nations audit. Open Forum Infect Dis 2018; 5: ofy313.

5. Levy MM, Artigas A, Phillips GS, Rhodes A, Beale R, Osborn $\mathrm{T}$, et al. Outcomes of the surviving sepsis campaign in intensive care units in the USA and Europe: a prospective cohort study. Lancet Infect Dis 2012; 12: 919-924.

6. Dellinger RP, Levy MM, Rhodes A, Annane D, Gerlach H, Opal SM, et al. Surviving sepsis campaign: international guidelines for management of severe sepsis and septic shock: 2012. Crit Care Med 2013; 41: 580-637.

7. Nardelli P, Senarighi G, Votta CD. Goal-Directed Therapy. In: Landoni G, Baiardo Redaelli M, Sartini C, Zangrillo A, Bellomo R, editors. Reducing Mortality in Critically Ill Patients. Cham: Springer International Publishing; 2021. p. 99-106.

8. Levy MM, Evans LE, Rhodes A. The surviving sepsis campaign bundle: 2018 update. Intensive Care Med 2018; 44: 925-928.

9. Wang L, Ma X, He H, Su L, Guo Y, Shan G, et al. Compliance with the Surviving Sepsis Campaign Guideline 1-hour bundle for septic shock in China in 2018. Ann Transl Med 2021; 9: 278.

10. Filbin MR, Thorsen JE, Zachary TM, Lynch JC, Matsushima M, Belsky JB, et al. Antibiotic delays and feasibility of a 1-hourfrom-triage antibiotic requirement: analysis of an emergency department sepsis quality improvement database. Ann Emerg Med 2020; 75: 93-99.

11. Seymour CW, Gesten F, Prescott HC, Friedrich ME, Iwashyna TJ, Phillips GS, et al. Time to treatment and mortality during mandated emergency care for sepsis. N Engl J Med 2017; 376: 2235-2244.

12. Peltan ID, Brown SM, Bledsoe JR, Sorensen J, Samore MH, Allen TL, et al. ED door-to-antibiotic time and long-term mortality in sepsis. Chest 2019; 155: 938-946.

13. Castańo P, Plaza M, Molina F, Hincapié C, Maya W, Cataño J, et al. Antimicrobial agent prescription: a prospective cohort study in patients with sepsis and septic shock. Trop Med Int Health 2019; 24: 175-184.
14. Abe T, Kushimoto S, Tokuda Y, Phillips GS, Rhodes A, Sugiyama $\mathrm{T}$, et al. Implementation of earlier antibiotic administration in patients with severe sepsis and septic shock in Japan: a descriptive analysis of a prospective observational study. Critical Care 2019; 23: 1-11.

15. Rothrock SG, Cassidy DD, Barneck M, Schinkel M, Guetschow B, Myburgh C, et al. Outcome of immediate versus early antibiotics in severe sepsis and septic shock: a systematic review and meta-analysis. Ann Emerg Med 2020; 76: 427-441.

16. Almutary A, Althunayyan S, Alenazi K, Alqahtani A, Alotaibi B, Ahmed M, et al. National Early Warning Score (NEWS) as prognostic triage tool for septic patients. Infect Drug Resist 2020; 13: 3843.

17. Shapiro SS, Wilk MB. An analysis of variance test for normality (complete samples). Biometrika 1965; 52: 591-611.

18. Mann HB, Whitney DR. On a Test of whether one of two random variables is stochastically larger than the other. Ann Math Stat 1947; 18: 50-60.

19. Freund Y, Khoury A, Möckel M, Karamercan M, Dodt C, Leach R, et al. European Society of Emergency Medicine position paper on the 1-hour sepsis bundle of the Surviving Sepsis Campaign: expression of concern. Eur J Emerg Med 2019; 26: 232-233.

20. Campaign SS. SSC hour-1 bundle updated. Surviving sepsis campaign. [Update 2019; Accessed 2021 January 11]. Available from: https://www.sccm.org/SurvivingSepsisCampaign/ Guidelines/Adult-Patients

21. IDSA Sepsis Task Force. Infectious Diseases Society of America (IDSA) POSITION STATEMENT: Why IDSA Did Not Endorse the Surviving Sepsis Campaign Guidelines. Clin Infect Dis 2018; 66: 1631-1635.

22. Sterling SA, Miller WR, Pryor J, Puskarich MA, Jones AE. The impact of timing of antibiotics on outcomes in severe sepsis and septic shock: a systematic review and meta-analysis. Crit Care Med 2015; 43: 1907.

23. Jalili $M$, Barzegari $H$, Pourtabatabaei N, Honarmand AR, Boreiri M, Mehrvarz A, et al. Effect of door-to-antibiotic time on mortality of patients with sepsis in emergency department: a prospective cohort study. Acta Medica Iranica 2013; 51: 454-460.

24. Whiles BB, Deis AS, Simpson SQ. Increased time to initial antimicrobial administration is associated with progression to septic shock in severe sepsis patients. Crit Care Med 2017; 45: 623-629.

25. Sherwin R, Winters ME, Vilke GM, Wardi G. Does early and appropriate antibiotic administration improve mortality in emergency department patients with severe sepsis or septic shock? J Emerg Med 2017; 53: 588-595. 